Canad. Math. Bull. Vol. 23 (3) 1980

\title{
THE RUIN PROBLEM FOR SUMS OF DEPENDENT RANDOM VARIABLES
}

\author{
BY \\ DUDLEY PAUL JOHNSON
}

\begin{abstract}
In this paper we show how to compute the probability that a sequence $Z_{n}=X_{0}+\cdots+X_{n}$ of partial sums of dependent random variables, each taking on the values \pm 1 , will first leave an interval $(a, b)$ at $a$; and how to compute the expected time it takes for the partial sums to leave the interval $(a, b)$.
\end{abstract}

Suppose that $M(\Omega, \mathscr{F})$ is the linear space of all measures $\varphi$ on the measurable space $(\Omega, \mathscr{F})$ of all functions $\omega$ mapping the non-negative integers into the finite set $\{-1,+1\}$ with $\mathscr{F}$ being the $\sigma$-field generated by the events $X_{t}(\omega)=$ $\omega(t)=\delta= \pm 1$; and that $\Phi$ is a linear subspace of $M(\Omega, \mathscr{F})$ which is closed under the operators $T$ and $E(\delta), \delta= \pm 1$, defined by

$$
\begin{gathered}
T \varphi\left(X_{0}=\delta_{0}, \ldots, X_{n}=\delta_{n}\right)=\varphi\left(X_{1}=\delta_{0}, \ldots, X_{n+1}=\delta_{n}\right) \\
E(\delta) \varphi(\Lambda)=\varphi\left(X_{0}=\delta, \Lambda\right) .
\end{gathered}
$$

Suppose that $a$ and $b$ are integers with $a<-1$ and $b>+1$ and that $\tau_{a b}(\omega)$ is the first time $n$ for which $\omega(0)+\cdots+\omega(n)$ is not in the interval $(a, b)$. Then we have the following

TheOrem. For each $\varphi \in \Phi$,

$$
\varphi\left[X_{0}+\cdots+X_{\tau_{a b}}=a\right]=p^{*} E(-1)\left[E(-1) A^{b-a-2} E(-1)\right]^{-1} A^{b-1} \varphi
$$

and

$$
\begin{aligned}
\int \tau_{a b}(\omega) \varphi(d \omega)=p^{*} B\left(I+\cdots+A^{b-2}\right) \varphi-p^{*} B(I & \left.+\cdots+A^{b-2}\right) \\
& \times\left[E(-1) A^{b-a-2} E(-1)\right]^{-1} A^{b-1} \varphi
\end{aligned}
$$

where the linear functional $p^{*}$ and the operators $A$ and $B$ are defined via

$$
\begin{aligned}
p^{*} \varphi & =\varphi(\Omega) \\
A & =[E(-1)+\lambda T E(+1)][\lambda E(+1)+T E(-1)]^{-1} \\
B & =[\lambda E(+1)-E(-1)][\lambda E(+1)+T E(-1)]^{-1}
\end{aligned}
$$

where it is assumed that a complex number $\lambda$ can be found such that the inverse of $\lambda E(+1)+T E(-1)$ exists and where $\left[E(-1) A^{b-a-2} E(-1)\right]^{-1}$ is the inverse of $E(-1) A^{b-a-2} E(-1)$ thought of as an operator mapping $E(-1) \Phi$ into $E(-1) \Phi$.

Received by the editors November 22, 1978 and, in revised form, Febuary 20, 1979. 
Proof. Let $f_{a, b}$ be the indicator function of the event $\left[X_{0}+\cdots+X_{\tau_{a, b}}=a\right]$ and let $f_{a, b}^{*}$ be the linear functional defined by $f_{a, b}^{*}=\int f_{a, b}(\omega) \varphi(d \omega)$. Then for any $\varphi \in \Phi$,

$$
\begin{aligned}
f_{a, b}^{*} T E(-1) \varphi & =f_{a-1, b-1}^{*} E(-1) \varphi \\
f_{a-1, b-1}^{*} T E(+1) \varphi & =f_{a, b}^{*} E(+1) \varphi \\
f_{-1, b}^{*} E(-1) \varphi & =p^{*} E(-1) \varphi \\
f_{a, 1}^{*} E(+1) \varphi & =0
\end{aligned}
$$

since if $\omega_{1}^{+}(u)=\omega(1+u)$ we have

$$
\begin{aligned}
f_{a, b}^{*} T \varphi & =\int f_{a, b}(\omega) T \varphi(d \omega) \\
& =\int f_{a, b}\left(\omega_{1}^{+}\right) \varphi(d \omega) \\
& =\int f_{a+\omega(0), b+\omega(0)}(\omega) \varphi(d \omega) \\
& =\int f_{a+\omega(0), b+\omega(0)}(\omega) E(-1) \varphi(d \omega)+\int f_{a+\omega(0), b+\omega(0)}(\omega) E(+1) \varphi(d \omega) \\
& =\int f_{a-1, b-1}(\omega) E(-1) \varphi(d \omega)+\int f_{a+1, b+1}(\omega) E(+1) \varphi(d \omega) \\
& =\int f_{a-1, b-1}^{*} E(-1) \varphi+f_{a+1, b+1}^{*} E(+1) \varphi
\end{aligned}
$$

which, upon replacing $\varphi$ by $E(-1) \varphi$ and $E(+1) \varphi$ give us equations (1) and (2). Equations (3) and (4) are obvious. From equations (1) and (2) we see that

$$
f_{a, b}^{*}[\lambda E(+1)+T E(-1)] \varphi=f_{a-1, b-1}^{*}[E(-1)+\lambda T E(+1)] \varphi
$$

or, in terms of the adjoints of these operators,

$$
[\lambda E(+1)+T E(-1)]^{*} f_{a, b}^{*}=[E(-1)+\lambda T E(+1)]^{*} f_{a-1, b-1}^{*}
$$

which now gives us

$$
f_{a, b}^{*}=[\lambda E(+1)+T E(-1)]^{*-1}[E(-1)+\lambda T E(+1)]^{*} f_{a-1, b-1}^{*}=A^{*} f_{a-1, b-1}^{*}
$$

or, by using equation (4) above,

$$
\begin{aligned}
f_{a, b}^{*}=A^{* b-1} f_{a-b+1,1}^{*} & =A^{* b-1} E(-1)^{*} f_{a-b+1,1}^{*}+A^{* b-1} E(+1)^{*} f_{a-b+1,1}^{*} \\
& =A^{* b-1} E(-1)^{*} f_{a-b+1,1}^{*} .
\end{aligned}
$$

Using equations (3) and (5), we see that

$$
E(-1)^{*} p^{*}=E(-1)^{*} f_{-1, b}^{*}=E(-1)^{*} A^{* b-1} E(-1)^{*} f_{-b, 1}^{*}
$$


so that

$$
E(-1)^{*} f_{-b, 1}^{*}=\left[E(-1)^{*} A^{* b-1} E(-1)^{*}\right]^{-1} E(-1)^{*} p^{*}
$$

where we take the inverse of $E(-1)^{*} A^{* b-1} E(-1)^{*}$ as an operator of $E(-1)^{*} \Phi^{*}$ into $E(-1)^{*} \Phi^{*}$. Thus by (5) and (6), with $-b$ replaced by $a-b+1$ in (6), we have

$$
f_{a, b}^{*} \varphi=A^{* b-1}\left[E(-1)^{*} A^{* b-a-2} E(-1)^{*}\right]^{-1} E(-1)^{*} p^{*} \varphi
$$

or

$$
\varphi\left[X_{0}+\cdots+X_{\tau_{a b}}=a\right]=p^{*} E(-1)\left[E(-1) A^{b-a-2} E(-1)\right]^{-1} A^{b-1} \varphi
$$

as was to be proved.

Now let $\tau_{a, b}^{*}$ be the linear functional on $\Phi$ defined by $\tau_{a, b}^{*} \varphi=\int \tau_{a, b}(\omega) \varphi(d \omega)$. Then the second part of the theorem will follow, as in the first part of the proof, from the equalities:

$$
\begin{aligned}
E(-1)^{*} T^{*} \tau_{a, b}^{*} & =E(-1)^{*} \tau_{a-1, b-1}^{*}-E(-1)^{*} p^{*} \\
E(+1)^{*} T^{*} \tau_{a-1, b-1}^{*} & =E(+1)^{*} \tau_{a, b}^{*}-E(+1)^{*} p^{*} \\
E(-1)^{*} \tau_{-1, b}^{*} & =0 \\
E(+1)^{*} \tau_{a,+1}^{*} & =0 .
\end{aligned}
$$

For example, the first two equalities follow from

$$
\begin{aligned}
T^{*} \tau_{a, b}^{*} \varphi & =\tau_{a, b}^{*} T \varphi \\
& =\int \tau_{a, b}(\omega) T \varphi(d \omega) \\
& =\int \tau_{a, b}\left(\omega_{1}^{+}\right) \varphi(d \omega) \\
& =\int \tau_{a, b}\left(\omega_{1}^{+}\right) E(-1) \varphi(d \omega)+\int \tau_{a, b}\left(\omega_{1}^{+}\right) E(+1) \varphi(d \omega) \\
& =\int\left[\tau_{a-1, b-1}(\omega)-1\right] E(-1) \varphi(d \omega)+\int\left[\tau_{a+1, b+1}(\omega)-1\right] E(+1) \varphi(d \omega) \\
& =\tau_{a-1, b-1}^{*} E(-1) \varphi+\tau_{a+1, b+1}^{*} E(+1) \varphi-p^{*} \varphi \\
& =E(-1)^{*} \tau_{a-1, b-1}^{*} \varphi+E(+1)^{*} \tau_{a+1, b+1}^{*} \varphi-p^{*} \varphi
\end{aligned}
$$

From (7) and (8) we now have

$$
\begin{aligned}
\tau_{a, b}^{*} & =A^{*} \tau_{a-1, b-1}^{*}+B^{*} p^{*}=\cdots \\
& =A^{* b-1} E(-1)^{*} \tau_{a-b+1,1}^{*}+\left(I^{*}+\cdots+A^{* b-2}\right) B^{*} p^{*}
\end{aligned}
$$

But by (9) and (11) we have

$$
0=E(-1)^{*} \tau_{-1, b}^{*}=E(-1)\left[A^{* b-1} E(-1)^{*} \tau_{-b, 1}^{*}+\left(I^{*}+\cdots+A^{* b-2}\right) B^{*} p^{*}\right]
$$


so that

$$
E(-1)^{*} \tau_{-b, 1}^{*}=-\left[E(-1)^{*} A^{* b-1} E(-1)^{*}\right]^{-1}\left[I^{*}+\cdots+A^{* b-2}\right] B^{*} p^{*} .
$$

Replacing $-b$ by $a-b+1$ in (12) and using (11) now gives us

$\tau_{a, b}^{*}=\left(I^{*}+\cdots+A^{* b-2}\right) B^{*} p^{*}-A^{* b-2}\left[E(-1)^{*} A^{* b-a-2} E(-1)^{*}\right]^{-1}\left(I^{*}+\cdots\right.$

or, equivalently,

$$
\begin{aligned}
\int \tau_{a b}(\omega) \varphi(d \omega)=p^{*} B\left(I+\cdots+A^{b-2}\right) \varphi-p^{*} B(I & \left.+\cdots+A^{b-2}\right) \\
& \times\left[E(-1) A^{b-a-2} E(-1)\right]^{-1} A^{b-1} \varphi
\end{aligned}
$$

which completes the proof of the theorem.

As an example, suppose that each probability measure $\varphi \in \Phi$ represents a temporally homogeneous Markov chain on the integers $\{-1,+1\}$. Then each $\varphi \in \Phi$ can be identified with its initial distribution so that $\Phi$ can be thought of as $R^{2}$ via $\varphi \rightarrow\left(\varphi\left(X_{0}=-1\right), \varphi\left(X_{0}=+1\right)\right)$. The operators $T^{*}, E(-1)^{*}, E(+1)^{*}$ and the linear functional $p^{*}$ now become, in matrix form, using $(1,0)$ and $(0,1)$ as a basis of $R^{2}$,

$$
T^{*}=\left(\begin{array}{ll}
p_{-1,-1} & p_{-1,+1} \\
p_{+1,-1} & p_{+1,+1}
\end{array}\right), \quad E(-1)^{*}=\left(\begin{array}{ll}
1 & 0 \\
0 & 0
\end{array}\right), \quad E(+1)^{*}=\left(\begin{array}{ll}
0 & 0 \\
0 & 1
\end{array}\right), \quad p^{*}=\left(\begin{array}{l}
1 \\
1
\end{array}\right) .
$$

In the case of the standard random walk, the measure $\varphi$ represents a sequence of independent, identically distributed random variables so that

$$
T^{*}=\left(\begin{array}{ll}
q & p \\
q & p
\end{array}\right), \quad q=\varphi\left(X_{k}=-1\right), \quad p=\varphi\left(X_{k}=+1\right) .
$$

In this latter case, the operator $A^{*}$ is given by

$$
A^{*}=\left[\lambda E(+1)^{*}+E(-1)^{*} T^{*}\right]^{-1}\left[E(-1)^{*}+\lambda E(+1)^{*} T^{*}\right]=q^{-1}\left(\begin{array}{cc}
1-p q & -p^{2} \\
q^{2} & p q
\end{array}\right)
$$

so that, after some computation, we have the well known result

$$
\begin{aligned}
\varphi\left(X_{0}+\cdots+X_{\tau_{a b}}=a\right)=A^{* b-1}\left[E(-1)^{*} A^{* b-a-2} E(-1)^{*}\right]^{-1} E(-1)^{*} p^{*} \varphi \\
= \begin{cases}\frac{\left(\frac{q}{p}\right)^{-1-a}-\left(\frac{q}{p}\right)^{b-a}}{1-\left(\frac{q}{p}\right)^{b-a}} \text { if } \varphi=(1,0), \text { i.e. } X_{0}=-1 \\
\frac{\left(\frac{q}{p}\right)^{1-a}-\left(\frac{q}{p}\right)^{b-a}}{1-\left(\frac{q}{p}\right)^{b-a}} \text { if } \varphi=(0,1), \text { i.e. } X_{0}=+1 .\end{cases}
\end{aligned}
$$




\section{REFERENCE}

1. Dunford, N. and Schwartz, S. (1958). Linear Operators I: General theory. Pure and Appl. Math., Vol. 7, Interscience, New York.

\section{Department of Mathematics}

UNIVERSITY OF CALGARY

2920, 24 AVENUE N.W.

Calgary Alberta T2N IN4 